Tôhoku Math. Journ.

24 (1972), 389-399.

\title{
ALMOST CONVERGENCE IN BANACH SPACES
}

\author{
J. C. KURTZ ${ }^{1}$
}

(Received Feb. 16, 1971; Revised Oct. 19, 1971)

1. Introduction and definitions. Suppose $X$ and $Y$ are Banach spaces, $Y$ being real, and $B=B[X, Y]$ is the space of all bounded linear operators from $X$ to $Y$. Let $Y^{+}$denote the weak sequential extension [9] of $Y$, considered as a subset of $Y^{* *}$. Unless otherwise specified, $Y^{+}$ will be taken with the norm topology of $Y^{* *}$. McWilliams [8] has shown that for a real Banach space $Y, Y^{+}$is norm closed in $Y^{* *}$, hence $Y^{+}$is also a Banach space. By a summability method from $X$ to $Y^{+}$we mean a matrix $A=\left(a_{n k}\right)$ of elements of $B$. We denote by $m(X), c(X)$ and $c_{0}(X)$ the spaces of $X$-valued bounded, convergent and null sequences, respectively. A notion of almost convergence for elements of $m(X)$ has been defined in [3]. It is shown there that a sequence $\chi=\left\{x_{n}\right\} \in m(X)$ is almost convergent if and only if $\left\{x^{*}\left(x_{n}\right)\right\}$ is almost convergent in the sense of Lorentz [7] for each $x^{*} \in X^{*}$. In general the limit is a point in $X^{* *}$, or more precisely, a point in $X^{+}$.

Definition 1. A method $A$ is conservative if for each $\chi=\left\{x_{n}\right\} \in c(x)$, $y_{n}=\sum_{k=0}^{\infty} a_{n k}\left(x_{k}\right)$ exists as a point in $Y^{+}$for each $n \geqq 0$, and $\left\{y_{n}\right\}$ is a convergent sequence in $Y^{+}$.

Definition 2. A method $A$ is almost conservative if for each $\chi=$ $\left\{x_{n}\right\} \in c(X), y_{n}=\sum_{k=0}^{\infty} a_{n k}\left(x_{k}\right)$ exists as a point in $Y^{+}$, and $\left\{y_{n}\right\}$ is an almost convergent sequence in $Y^{+}$.

Definition 3. A method $A$ is regular relative to an operator $L \in B\left[X, Y^{+}\right]$if for each $\chi=\left\{x_{n}\right\} \in c(X)$ with $\lim _{n \rightarrow \infty} x_{n}=x, y_{n}=\sum_{k=0}^{\infty} a_{n k}\left(x_{k}\right)$ exists as a point in $Y^{+}$for each $n \geqq 0$, and $\lim _{n \rightarrow \infty} y_{n}=L(x)$ in $Y^{+}$.

Definition 4. A method $A$ is almost regular relative to an operator $L \in B\left[X, Y^{+}\right]$if for each $\chi=\left\{x_{n}\right\} \in c(X)$ with $\lim _{n \rightarrow \infty} x_{n}=x, y_{n}=\sum_{k=0}^{\infty} a_{n k}\left(x_{k}\right)$ exists as a point in $Y^{+}$, and $\left\{y_{n}\right\}$ is almost convergent in $Y^{+}$to $L(x)$.

In section 2 we give characterizations of conservative and almost conservative methods. In the first case we obtain a general form of the

1) I am indebted to the referee for his careful attention to detail, and his helpful suggestions. 
classical Toeplitz theorem. It should be noted that our version differs from that given by Kurtz and Tucker [5] in that we have chosen the norm topology in $Y^{+}$, while they have given $Y^{+}$the weak* topology from $Y^{* *}$. In the second case we obtain a more general form of the result given by King [2]. In section 3 a result is given for a type of strong regularity similar to that considered by Lorentz [7] in the scalar case. Hausdorff methods are considered in section 4 , and in section 5 the preceding results are used to give a stronger form of the meanergodic theorem.

\section{Conservative and almost conservative methods.}

THEOREM 1. The method $A$ is almost conservative if and only if

(i ) $\left\|\sum_{k=0}^{m} a_{n k}\left(x_{k}\right)\right\|_{Y} \leqq M \sup _{k}\left\|x_{k}\right\|$ for all $m, n \geqq 0$ and each $\left\{x_{k}\right\} \in m(X)$. $x \in X$.

(ii) $a_{n k}(x)$ almost converges $(n \rightarrow \infty)$ in $Y^{+}$to $c_{k}(x)$ for each $k \geqq 0$,

(iii) $\left\{\sum_{k=0}^{\infty} a_{n k}(x)\right\}$ is almost convergent in $Y^{+}$for each $x \in X$. We call this limit $L(x)$.

We first observe that condition (i) is equivalent to

(i ) $\quad\left\|\sum_{k=0}^{\infty} a_{n k}\left(x_{k}\right)\right\|_{Y^{+}} \leqq M \sup _{k}\left\|x_{k}\right\|$ for all $n \geqq 0$

and each $\left\{x_{k}\right\} \in m(X)$, the series being weakly convergent in $Y$. This equivalence has been shown in [5].

Lemma 1. Condition (i) implies $\left\|a_{n k}\right\|_{B} \leqq M$ for all $n, k \geqq 0$.

Lemma 2. Conditions (i) and (ii) imply $c_{k} \in B\left[X, Y^{+}\right]$.

Proof. Take $y^{*} \in Y^{*}$, then

$$
\begin{aligned}
\left\|c_{k}(x)\right\|_{Y^{+}} & =\sup _{\left\|y^{*}\right\| \leq 1}\left|\lim _{p \rightarrow \infty} y^{*}\left\{\frac{a_{n k}(x)+\cdots+a_{n+p, k}(x)}{p+1}\right\}\right| \\
& \leqq \sup _{\left\|y^{*}\right\| \leq 1} \varlimsup_{p \rightarrow \infty}\left\|y^{*}\right\|\left\|\frac{a_{n k}(x)+\cdots+a_{n+p, k}(x)}{p+1}\right\| \\
& \leqq M\|x\| .
\end{aligned}
$$

Lemma 3. Conditions (i) and (ii) imply $\sum_{k=0}^{\infty} c_{k}\left(x_{k}\right)$ converges in $Y^{+}$ for each $\left\{x_{k}\right\} \in c_{0}(x)$.

Proof. Given $\varepsilon>0$, choose $N>0$ such that $k \geqq N$ implies $\left\|x_{k}\right\|<$ $\varepsilon / M$. Take $y_{1}^{*}, y_{2}^{*} \in Y^{*}$ and set $\varepsilon_{k}=\operatorname{sgn} y_{1}^{*} c_{k}\left(x_{k}\right)$. 


$$
\begin{aligned}
\sum_{k=r}^{s}\left|y_{1}^{*}\left(c_{k}\left(x_{k}\right)\right)\right| & =\left|y_{1}^{*} \sum_{k=r}^{s} c_{k}\left(\varepsilon_{k} x_{k}\right)\right| \\
& \leqq\left\|y_{1}^{*}\right\| \sup _{\left\|y_{2}\right\|_{1} \| \leqq}\left|\lim _{p \rightarrow \infty} y_{2}^{*} \sum_{k=r}^{s}\left\{\frac{a_{n k}\left(\varepsilon_{k} x_{k}\right)+\cdots+a_{n+p, k}\left(\varepsilon_{k} x_{k}\right)}{p+1}\right\}\right| \\
& \leqq\left\|y_{1}^{*}\right\| \varlimsup_{p \rightarrow \infty} \frac{1}{p+1} \sum_{i=0}^{p}\left\|\sum_{k=r}^{s} a_{n+i, k}\left(\varepsilon_{k} x_{k}\right)\right\| \\
& \leqq\left\|y_{1}^{*}\right\| M \sup _{k \geq r}\left\|x_{k}\right\| .
\end{aligned}
$$

If we take $r \geqq N$ we have $\left\|\sum_{k=r}^{s} c_{k}\left(x_{k}\right)\right\|_{Y^{+}}<\varepsilon$, and since $Y^{+}$is complete, $\sum_{k=0}^{\infty} c_{k}\left(x_{k}\right)$ exists in $Y^{+}$.

Lemma 4. Conditions (i) and (iii) imply $L \in B\left[X, Y^{+}\right]$.

Now consider an element $\chi=\left\{x_{n}\right\} \in c(X)$, and suppose $\lim _{n \rightarrow \infty} x_{n}=x$. We will use the notation $\left\{\widetilde{x}_{n}\right\}=\left(0, \cdots,,_{n}^{(n)}, 0, \cdots\right)$ and $\{x\}=(x, x, \cdots)$. Exactly as in the classical case one can prove the

LEMMA 5. $\chi=\sum_{k=0}^{\infty}\left\{\widehat{x_{k}-x}\right\}+\{x\}$, the convergence being in $c(X)$.

Proof of Theorem 1. For the sufficiency, suppose $A$ satisfies the conditions (i), (ii) and (iii), and consider $\chi \in c(X)$. Then we have $\chi=$ $\sum_{k=0}^{\infty}\left\{x_{k}-x\right\}+\{x\}$ where $x=\lim _{n \rightarrow \infty} x_{n}$. Set

$$
t_{n p}(\chi)=\frac{1}{p+1} \sum_{j=n}^{n+p} \sum_{k=0}^{\infty} a_{j k}\left(x_{k}\right)
$$

For each $z^{*} \in\left(Y^{+}\right)^{*}$ we have

$$
\begin{aligned}
\left|z^{*} t_{n p}(\chi)\right| & \leqq \frac{\left\|z^{*}\right\|}{p+1} \sum_{j=n}^{n+p}\left\|\sum_{k=0}^{\infty} a_{j k}\left(x_{k}\right)\right\|_{Y^{+}} \\
& \leqq\left\|z^{*}\right\| M \sup _{k}\left\|x_{k}\right\|
\end{aligned}
$$

so that $z^{*} t_{n p} \in c(X)^{*}$ for all $n, p \geqq 0$, and

$$
z^{*} t_{n p}(\chi)=\sum_{k=0}^{\infty} z^{*} t_{n p}\left(\widehat{\left(x_{k}-x\right\}}\right)+z^{*} t_{n p}(\{x\}) \text {. }
$$

Conditions (ii) and (iii) imply $\lim _{p \rightarrow \infty} z^{*} t_{n p}(\chi)=z^{*} t_{n}(\chi)$ exists for a fundamental set in $c(X)$, hence for all $\chi \in c(X)$. Define

$$
t(\chi)=\sum_{k=0}^{\infty} c_{k}\left(x_{k}-x\right)+L(x) \text {. }
$$

Given $\varepsilon>0$, choose $N>0$ such that $\sup _{k \geqq N+1}\left\|x_{k}-x\right\|<\varepsilon$ and

$$
\left\|\sum_{k=N+1}^{\infty} c_{k}\left(x_{k}-x\right)\right\|_{Y^{+}}<\varepsilon .
$$


Then there exists $N_{1}$ such that $p \geqq N_{1}$ implies

$$
\begin{aligned}
\left|z^{*} t_{n p}(\chi)-z^{*} t(\chi)\right| \leqq & \left|z^{*} t_{n p}(\{x\})-z^{*} L(x)\right| \\
& +\sum_{k=0}^{N}\left|z^{*} t_{n p}\left(\widehat{\left.x_{k}-x\right\}}\right)-z^{*} c_{k}\left(x_{k}-x\right)\right| \\
& +\left\|z^{*}\right\|\left\{M \sup _{k \leqq N+1}\left\|x_{k}-x\right\|+\left\|\sum_{k=N+1}^{\infty} c_{k}\left(x_{k}-x\right)\right\|_{Y^{+}}\right\} \\
\leqq & \varepsilon\left(1+\left\|z^{*}\right\|(M+1)\right) \quad \text { for all } n \geqq 0 .
\end{aligned}
$$

Therefore $\left\{\sum_{k=0}^{\infty} a_{n k}\left(x_{k}\right)\right\}$ is almost convergent in $Y^{+}$to

$$
L(x)+\sum_{k=0}^{\infty} c_{k}\left(x_{k}-x\right) .
$$

The necessity of conditions (ii) and (iii) follows immediately by considering the sequences $(x, x, \cdots)$ and $(0,0, \cdots, x, 0, \cdots)$ for each $x \in X$. For condition (i) we consider any $\chi \in c(X)$ and set $T_{m n}(\chi)=\sum_{k=0}^{m} a_{n k}\left(x_{k}\right)$. Then $T_{m n} \in B\left[c(X), Y^{* *}\right]$, and since $A$ is almost conservative, $\left\{T_{m n}(\chi)\right\}$ is $w^{*}$ convegent in $Y^{* *}$, hence bounded and therefore there exist numbers $M_{n}$ such that $\left\|\sum_{k=0}^{m} a_{n k}\left(x_{k}\right)\right\|_{Y} \leqq M_{n} \sup _{k}\left\|x_{k}\right\|$. If we denote $\lim _{m \rightarrow \infty} y^{*} T_{m n}(\chi)=$ $y^{*} T_{n}(\chi)$ then $T_{n} \in B\left[c(X), Y^{+}\right]$and $\left\|T_{n}\right\| \leqq M_{n}$. Since $\left\{T_{n}(\chi)\right\}$ is almost convergent in $Y^{+}$it is bounded, and hence there exists $M$ such that $\left\|T_{n}\right\|_{B\left[c(X), Y^{+}\right]} \leqq M$. If we truncate $\chi$ at $k=m$ we have the condition

$$
\left\|T_{n}(\chi)\right\|_{Y^{+}}=\left\|\sum_{k=0}^{m} a_{n k}\left(x_{k}\right)\right\|_{Y} \leqq M \sup _{k}\left\|x_{k}\right\|
$$

which is (i).

COROLLARY 1. A is almost regular relative to a linear operator $L$ from $X$ to $Y^{+}$if and only if conditions (i), (ii) and (iii) hold with $c_{k}(x)=\theta$ for all $k \geqq 0, x \in X$.

THEOREM 2. The method $A$ is conservative if and only if

(i) $\left\|\sum_{k=0}^{m} a_{n k}\left(x_{k}\right)\right\|_{r} \leqq M \sup _{k}\left\|x_{k}\right\|$ for all $m, n \geqq 0$ and each $\left\{x_{k}\right\} \in m(X)$

(ii) $a_{n k}(x)$ converges $(n \rightarrow \infty)$ in $Y^{+}$to $c_{k}(x)$ for each $k \geqq 0, x \in X$

(iii) $\left\{\sum_{k=0}^{\infty} a_{n k}(x)\right\}$ is convergent in $Y^{+}$for each $x \in X$.

We call the limit $L(x)$.

Proof. For the sufficiency, one can easily show (see Lemma 3 ) that for $\chi \in c_{0}(X)$, both $\sum_{k=0}^{\infty} a_{n k}\left(x_{k}\right)$ and $\sum_{k=0}^{\infty} c_{k}\left(x_{k}\right)$ converge in norm. If $\chi \in c(X)$ then we set $T_{n}(\chi)=\sum_{k=0}^{\infty} a_{n k}\left(x_{k}-x\right)+\sum_{k=0}^{\infty} a_{n k}(x)$, where $x=\lim _{n \rightarrow \infty} x_{n}$, and $T(\chi)=\sum_{k=0}^{\infty} c_{k}\left(x_{k}-x\right)+L(x)$. Then we have 


$$
\begin{aligned}
\left\|T_{n}(\chi)-T(\chi)\right\| \leqq & \left\|\sum_{k=0}^{\infty} a_{n k}(x)-L(x)\right\| \\
& +\sum_{k=0}^{N}\left\|a_{n k}\left(x_{k}-x\right)-c_{k}\left(x_{k}-x\right)\right\| \\
& +\left\|\sum_{k=N+1}^{\infty} a_{n k}\left(x_{k}-x\right)\right\| \\
& +\left\|\sum_{k=N+1}^{\infty} c_{k}\left(x_{k}-x\right)\right\|
\end{aligned}
$$

and the conclusion follows at once. The necessity follows as in Theorem 1.

COROLlaRY 2. A is regular relative to a linear operator $L$ from $X$ to $Y^{+}$if and only if conditions (i), (ii)' and (iii)' hold with $c_{k}(x)=\theta$ for all $k \geqq 0, x \in X$.

3. Strong regularity. We are unable at this time to give a complete analogue to Lorentz' characterization [7] of strong regularity, but the following partial result proves to be useful.

THeOREM 3. Suppose $A$ is regular relative to $L \in B\left[X, Y^{+}\right]$, and for each $\varepsilon>0$ there exists $N>0$ such that

$$
\left\|\sum_{k=0}^{\infty}\left(a_{n k}-a_{n, k+1}\right) x_{k}\right\|_{Y^{+}} \leqq \varepsilon \sup _{k}\left\|x_{k}\right\|
$$

for all $n \geqq N$ and $\chi \in m(X)$. If $\chi \in m(X)$ is such that

$$
\lim _{p \rightarrow \infty}\left\|\frac{x_{n}+\cdots+x_{n+p}}{p+1}-x\right\|=0
$$

uniformly in $n$, then

$$
\lim _{n \rightarrow \infty}\left\|\sum_{k=0}^{\infty} a_{n k}\left(x_{k}\right)-L(x)\right\|_{Y^{+}}=0
$$

Proof. Given $\varepsilon>0$, choose $p_{0}$ such that

$$
\frac{1}{p+1}\left(x_{n}+\cdots+x_{n+p}\right)=x+\alpha_{n p}
$$

where $\left\|\alpha_{n p}\right\| \leqq \varepsilon$ for $p \geqq p_{0}, n \geqq 0$. Fix $p \geqq p_{0}$. We then have

$$
\frac{1}{p+1} \sum_{n=0}^{\infty} a_{m n}\left(x_{n}+\cdots+x_{n+p}\right)=\sum_{n=0}^{\infty} a_{m n}(x)+\sum_{n=0}^{\infty} a_{m n}\left(\alpha_{n p}\right) \text {. }
$$

A short calculation yields the formula 


$$
\begin{aligned}
\sum_{n=0}^{\infty} a_{m n}\left(x_{n}\right)-L(x)= & \sum_{n=0}^{\infty} a_{m n}(x)-L(x) \\
& +\sum_{n=0}^{\infty} a_{m n}\left(\alpha_{n p}\right)+\sum_{n=0}^{p-1} a_{m n}\left(x_{n}\right)-\frac{1}{p+1} \sum_{i=0}^{p-1} \sum_{n=0}^{p-1-i} a_{m n}\left(x_{n+i}\right) \\
& -\frac{1}{p+1} \sum_{n=p}^{\infty} \sum_{\rho=0}^{p} \sum_{k=n-\rho}^{n-1}\left(a_{m k}-a_{m, k+1}\right) x_{n} .
\end{aligned}
$$

This yields the estimate

$$
\begin{aligned}
\left\|\sum_{n=0}^{\infty} a_{m n}\left(x_{n}\right)-L(x)\right\|_{Y^{+}} \leqq & \left\|\sum_{n=0}^{\infty} a_{m n}(x)-L(x)\right\|_{Y^{+}} \\
& +M \varepsilon+\sum_{n=0}^{p-1}\left\|a_{m n}\left(x_{n}\right)\right\|_{Y^{+}} \frac{1}{p+1} \sum_{i=0}^{p-1} \sum_{n=0}^{p-1-i}\left\|a_{m n}\left(x_{n+i}\right)\right\|_{Y} \\
& +\frac{1}{p+1} \sum_{\rho=0}^{p}\left\|\sum_{k=0}^{\infty}\left(a_{m k}-a_{m, k+1}\right) z_{k \rho}\right\|_{Y^{+}}
\end{aligned}
$$

where

$$
z_{k \rho}=\left\{\begin{array}{cl}
0 & ; \quad 0 \leqq k \leqq p-\rho-1 \\
\sum_{n=p}^{k+\rho} x_{n} & ; \quad p-\rho \leqq k \leqq p-2 \\
\sum_{n=k+1}^{k+\rho} ; & k \geqq p-1
\end{array}\right.
$$

and $\sup _{k}\left\|z_{k \rho}\right\| \leqq \rho \sup _{n}\left\|x_{n}\right\|$.

Now choose $N$ such that $m \geqq N$ implies

$$
\left\|\sum_{k=0}^{\infty}\left(a_{m k}-a_{m, k+1}\right) x_{k}\right\|_{Y^{+}} \leqq \frac{2 \varepsilon}{p} \sup _{k}\left\|x_{k}\right\| \text {. }
$$

Then there exists $N_{1} \geqq N$ such that $m \geqq N_{1}$ implies

$$
\begin{aligned}
\left\|\sum_{n=0}^{\infty} a_{m n}\left(x_{n}\right)-L(x)\right\|_{Y^{+}} \leqq & \varepsilon+M \varepsilon+M+2 \varepsilon \sup _{n}\left\|x_{n}\right\| \\
& +\frac{2 \varepsilon}{p(p+1)} \sum_{\rho=0}^{p} \rho \sup \left\|x_{n}\right\| \\
\leqq & \left(1+M+3 \sup _{n}\left\|x_{n}\right\|\right) \varepsilon .
\end{aligned}
$$

The conclusion follows.

4. Hausdorff methods. Consider now a Hausdorff method $H(\mu)$ defined by setting $a_{n k}=\left(\begin{array}{l}n \\ k\end{array}\right) \Delta^{n-k} \mu_{k}$, where $\left\{\mu_{n}\right\} \subseteq B[X, Y]$. Suppose (i) is satisfied, i.e., $\left\|\sum_{k=0}^{n}\left(\begin{array}{l}n \\ k\end{array}\right) \Delta^{n-k} \mu_{k}\left(x_{k}\right)\right\|_{Y} \leqq M \sup _{k}\left\|x_{k}\right\|$ for all $n \geqq 0$, $\chi \in m(X)$. Conditions (iii) and (iii)' are then automatically satisfied since 
$\sum_{k=0}^{n}\left(\begin{array}{l}n \\ k\end{array}\right) \Delta^{n-k} \mu_{k}(x) \equiv \mu_{0}(x)$. In [5] it is shown that (i) is equivalent to the existence of a function $K(t) \in B\left[X, Y^{+}\right]$for $t \in[0,1]$ such that $K$ satisfies the Gowurin $w$-property [1] and $\mu_{n}(x)=\int_{0}^{1} d K(t) \cdot\left(t^{n} x\right)$ for all $n \geqq 0, x \in X$, the integral converging in $Y^{+}$norm. Using the same method as in [5] one can show that $a_{n k}(x)$ converges weakly in $Y^{+}(n \rightarrow \infty)$ for each $k$, hence condition (ii) is also satisfied. As a result, $H(\mu)$ is almost conservative if and only if condition (i) holds. It has been pointed out by D.H. Tucker (private communication) that condition (ii)' does not follow automatically from condition (i) as in the scalar case. However, if we assume that the generating function $K(t)$ is continuous at $t=0$, then we have

$$
\begin{aligned}
\left\|a_{n k}(x)\right\|_{Y^{+}} \leqq & \left(\begin{array}{l}
n \\
k
\end{array}\right)\left\|\int_{0}^{\delta} d K(t) \cdot\left\{t^{k}(1-t)^{n-k} x\right\}\right\|_{Y^{* *}} \\
& +\left(\begin{array}{l}
n \\
k
\end{array}\right)\left\|\int_{\delta}^{1} d K(t) \cdot\left\{t^{k}(1-t)^{n-k} x\right\}\right\|_{Y^{* *}} \\
\leqq & \left(\begin{array}{l}
n \\
k
\end{array}\right)\left(\frac{k}{n}\right)^{k}\left(1-\frac{k}{n}\right)^{n-k} W_{0}^{\delta} K \cdot\|x\| \\
& +\left(\begin{array}{l}
n \\
k
\end{array}\right) \delta^{k}(1-\delta)^{n-k} W_{0}^{1} K \cdot\|x\|
\end{aligned}
$$

where the first term can be made small independent of $n$ by choosing $\delta$ small, and the second term tends to zero $(n \rightarrow \infty)$ for $\delta$ fixed, and it follows that (ii)' is satisfied.

Now suppose $H(\mu)$ is regular relative to $L \in B\left[X, Y^{+}\right]$and $\mu_{n}(x)=$ $\int_{0}^{1} d K(t) \cdot\left(t^{n} x\right)$.

THEOREM 4. If $K(t)$ is continuous at $t=0$ and $t=1$, then $H(\mu)$ satisfies the condition $(*)$ of Theorem 3.

Lemma 6. If $\delta>0$ is fixed, then

$$
\lim _{n \rightarrow \infty} \sup _{\delta \leq t \leq 1-\delta} \sum_{k=0}^{n}\left|\left(\begin{array}{l}
n \\
k
\end{array}\right) t^{k}(1-t)^{n-k}-\left(\begin{array}{c}
n \\
k+1
\end{array}\right) t^{k+1}(1-t)^{n-k-1}\right|=0 .
$$

ProOF.

$$
\begin{aligned}
& \sum_{k=0}^{n}\left|\left(\begin{array}{l}
n \\
k
\end{array}\right) t^{k}(1-t)^{n-k}-\left(\begin{array}{c}
n \\
k+1
\end{array}\right) t^{k+1}(1-t)^{n-k-1}\right| \\
= & \frac{1}{1-t} \sum_{k=0}^{n}\left(\begin{array}{l}
n \\
k
\end{array}\right) t^{k}(1-t)^{n-k} \frac{n+1}{k+1}\left|\frac{k+1}{n+1}-t\right| .
\end{aligned}
$$


We split the sum according to the sets

$$
A=\left\{k:\left|t-\frac{k+1}{n+1}\right|<\alpha\right\} \quad \text { and } \quad B=\left\{k:\left|t-\frac{k+1}{n+1}\right| \geqq \alpha\right\}
$$

where $\alpha>0$.

$$
\begin{aligned}
\left|\sum_{A}\right| & \leqq \frac{\alpha}{1-t} \sum_{k=0}^{n}\left(\begin{array}{l}
n \\
k
\end{array}\right) \frac{n+1}{k+1} t^{k}(1-t)^{n-k} \\
& \leqq \frac{\alpha}{t(1-t)} \sum_{k=0}^{n+1}\left(\begin{array}{c}
n+1 \\
k
\end{array}\right) t^{k}(1-t)^{(n+1)-k} \\
& =\frac{\alpha}{t(1-t)} \leqq \frac{\alpha}{\delta(1-\delta)} .
\end{aligned}
$$

In the second sum we use the estimate

$$
\begin{aligned}
&\left|t-\frac{k+1}{n+1}\right| \leqq 1 \leqq \frac{\left(t-\frac{k+1}{n+1}\right)^{2}}{\alpha^{2}} \\
&\left|\sum_{B}\right| \leqq \frac{1}{\alpha^{2}(1-t)} \sum_{k=0}^{n}\left(\begin{array}{c}
n+1 \\
k+1
\end{array}\right) t^{k}(1-t)^{n-k}\left(t-\frac{k+1}{n+1}\right)^{2} \\
& \leqq \frac{1}{\alpha^{2}(n+1)^{2} t(1-t)} \sum_{k=0}^{n+1}\left(\begin{array}{c}
n+1 \\
k
\end{array}\right) t^{k}(1-t)^{(n+1)-k}[k-(n+1) t]^{2} \\
&=\frac{1}{\alpha^{2}(n+1)} .
\end{aligned}
$$

Given $\varepsilon>0$ choose $\alpha$ such that $\alpha /(\delta(1-\delta))<\varepsilon / 2$. Then $n \geqq 2 /\left(\alpha^{2} \varepsilon\right)-1$ implies

$$
\sum_{k=0}^{n}\left|\left(\begin{array}{l}
n \\
k
\end{array}\right) t^{k}(1-t)^{n-k}-\left(\begin{array}{c}
n \\
k+1
\end{array}\right) t^{k}(1-t)^{n-k-1}\right|<\frac{\varepsilon}{2}+\frac{\varepsilon}{2}=\varepsilon .
$$

Proof of Theorem 4. Take $\chi=\left\{x_{k}\right\} \in m(X)$.

$$
\begin{aligned}
\left\|\sum_{k=0}^{n}\left(a_{n k}-a_{n, k+1}\right) x_{k}\right\| & \|\| \int_{0}^{1} d K(t) \sum_{k=0}^{n}\left\{\left(\begin{array}{l}
n \\
k
\end{array}\right) t^{k}(1-t)^{n-k}-\left(\begin{array}{c}
n \\
k+1
\end{array}\right) t^{k+1}(1-t)^{n-k-1}\right\} x_{k} \| \\
\leqq & \left\|\int_{0}^{\delta}\right\|_{Y^{* *}}+\left\|\int_{\delta}^{1-\delta}\right\|\left\|_{Y^{* *}}+\right\| \int_{1-\delta}^{1}\|\|_{Y^{* *}} \\
\leqq & \left\{2 W_{0}^{\delta} K+2 W_{1-\delta}^{1} K+W_{0}^{1} K \sup _{\delta \leqq t \leqq 1-\delta} \sum_{k=0}^{n} \mid \begin{array}{l}
n \\
k
\end{array}\right) t^{k}(1-t)^{n-k} \\
& \left.-\left(\begin{array}{c}
n \\
k+1
\end{array}\right) t^{k+1}(1-t)^{n-k-1} \mid\right\} \cdot \sup _{k}\left\|x_{k}\right\| .
\end{aligned}
$$


The conclusion follows now from the continuity of the Gowurin constant at $t=0$ and $t=1$, and Lemma 6 .

5. The ergodic theorem. Now let $X$ be a reflexive Banach space. In this context Lorch [6] has proved that if $V \in B[X]$ such that $\left\|V^{n}\right\| \leqq C$ for all $n \geqq 0$, then the sequence $\left\{V^{n}\right\}$ is pointwise $(C, 1)$-summable in $X$ to $P$, the projection onto the null space of $(I-V)$. We first extend this result.

THEOREM 5. If $V \in B[X]$ such that $\left\|V^{n}\right\| \leqq C$ for all $n \geqq 0$, then

$$
\lim _{p \rightarrow \infty}\left\|\frac{V^{n}(x)+\cdots+V^{n+p}(x)}{p+1}-P(x)\right\|_{X}=0
$$

uniformly in $n$ for each $x \in X$.

Proof. Let $N=\operatorname{ker}(I-V)$ and $R=$ closure of the range of $(I-V)$. Set $T_{n p}=1 /(p+1)\left(V^{n}+\cdots+V^{n+p}\right)$. If $x \in N$ then $T_{n p}(x)=x$. If $x \in R$, then there exist $y, z \in X$ such that $x=(y-V y)+z$ with $\|z\|<\varepsilon$. We then have

$$
T_{n p}(x)=\frac{V^{n}}{p+1}\left\{\left(y-V^{p+1} y\right)+\left(z+V z+\cdots+V^{p} z\right)\right\}
$$

hence

$$
\left\|T_{n p}(x)\right\| \leqq \frac{2 C^{2}\|y\|}{p+1}+C^{2} \varepsilon \quad \text { (assume } C \geqq 1 \text { ) }
$$

so that $\lim _{p \rightarrow \infty} T_{n p}(x)=0$ uniformly in $n$. The remaining details follow as in Lorch [6].

COROLLARY 3. Let $H(\mu)$ be a Hausdorff method regular with respect to $L \in B[X, Y]$ with generating function $K(t)$ continuous at $t=0$ and $t=1$. Then

$$
\lim _{n \rightarrow \infty}\left\|\sum_{k=0}^{n}\left(\begin{array}{l}
n \\
k
\end{array}\right) \Delta^{n-k} \mu_{k}\left\{V^{k}(x)\right\}-L P(x)\right\|_{Y}=0
$$

for each $x \in X$.

Proof. Apply Theorems 3, 4 and 5.

We should note here that the convergence is in $Y$ rather than $Y^{+}$ since $L \in B[X, Y]$ by assumption. This corollary has been proved directly by Kurtz and Tucker [4]. They assume a weaker form of regularity which is equivalent to (i) (their condition A), however the assumption that $K(t)$ is continuous at $t=0$ implies our stronger type of regularity, 
as was noted in section 4. The condition of continuity of $K(t)$ at $t=1$ becomes transparent from this point of view also when one remembers that in the scalar case continuity of the generating function at $t=1$ is necessary and sufficient for strong regularity [7]. Our methods also yield the stronger

THEOREM 6. If $V \in B[X]$ such that $\left\|V^{n}\right\| \leqq C$ for all $n \geqq 0, A$ is a method regular with respect to $L \in B\left[X, Y^{+}\right]$, and $A$ satisfies condition (*) of Theorem 3, then

$$
\lim _{n \rightarrow \infty}\left\|\sum_{k=0}^{\infty} a_{n k}\left\{V^{k}(x)\right\}-L P(x)\right\|_{Y^{+}}=0
$$

for each $x \in X$.

Proof. Apply Theorems 3 and 5.

CoRollary 4. If $X=Y$ and $A$ is a strongly regular scalar method, then

$$
\lim _{n \rightarrow \infty}\left\|\sum_{k=0}^{\infty} a_{n k} V^{k}(x)-P(x)\right\|_{X}=0
$$

for each $x \in X$.

Proof. If $\chi \in m(X)$ then

$$
\left\|\sum_{k=0}^{\infty}\left(a_{n, k}-a_{n, k+1}\right) x_{k}\right\|_{X} \leqq \sup _{k}\left\|x_{k}\right\| \sum_{k=0}^{\infty}\left|a_{n k}-a_{n, k+1}\right|
$$

hence $(*)$ is satisfied. The result follows.

\section{BIBLIOGRAPHY}

[1] M. GowURIN, Über die Stieltjesche Integration abstrakter Functionen, Fund. Math. 27 (1936), 254-268.

[2] J. P. KING, Almost summable sequences, Proc. Amer. Math. Soc. 17 (1966), 1219-1225.

[3] J. C. KURtz, Almost convergent vector sequences, Tôhoku Math. Journ. 22 (1970).

[4] L. C. Kurtz AND D. H. TUCKer, An extended form of the mean-ergodic theorem, Pacific Jour. Math. 27 (1968), 539-545.

[5] — Vector-valued summability methods on a linear normed space, Proc. Amer. Math. Soc. 16 (1965), 419-428.

[6] E. R. LORCH, Spectral Theory, Oxford University Press, New York, 1962.

[7] G. G. LoRentz, A contribution to the theory of divergent sequences, Acta Math. 80 (1948), 167-190.

[8] R. D. McWilliams, A note on weak sequential convergence, Pac. Jour. Math. 12 (1963), 333-335.

[9] D. H. TUCKER, A representation theorem for a continuous linear transformation on a space of continuous functions, Proc. Amer. Math. Soc. 16 (1965), 946-953. 


\section{ALMOST CONVERGENCE IN BANACH SPACES}

[10] K. Zeller, Verallgemeinerte Matrixtransformation, Math. Zeit. 56 (1952), 18-20.

Department of Mathematics

Michigan State University

East Lansing, Michigan

U.S.A. 
